\title{
ERRATUM
}

\section{Erratum to: Exploring the Interplay between Rescue Drugs, Data Imputation, and Study Outcomes: Conceptual Review and Qualitative Analysis of an Acute Pain Data Set}

Neil K. Singla $\cdot$ Diana S. Meske $\cdot$ Paul J. Desjardins

Published online: August 29, 2017

(c) The Author(s) 2017. This article is an open access publication

Erratum to: Pain Ther

DOI 10.1007/s40122-017-0074-5

The reference citations in Table 1 were published incorrectly in the original publication.

The updated Table 1 is provided below: under doi:10.1007/s40122-017-0074-5.

N. K. Singla $(\bowtie)$

Lotus Clinical Research, Huntington Hospital, Department of Anesthesiology, Pasadena, CA, USA

e-mail: nsingla@lotuscr.com

D. S. Meske

Collegium Pharmaceutical, Inc, Canton, MA, USA

A9 P. J. Desjardins

A10 Desjardins Associates, LLC, Maplewood, NJ, USA 
Table 1 Description of studies included in analysis

\begin{tabular}{|c|c|c|c|c|c|c|}
\hline Study & Study drug & Rescue regimen & $\begin{array}{l}\text { Rescue } \\
\text { usage }^{a}\end{array}$ & $\begin{array}{l}\text { Pain scale } \\
\text { used }\end{array}$ & $\begin{array}{l}\text { Placebo } \\
\text { group N } \mathbf{N}^{\mathrm{b}}\end{array}$ & $\begin{array}{l}\text { Study drug } \\
\text { group } N^{b, c}\end{array}$ \\
\hline $\begin{array}{l}\text { Daniels Upmalis } 2009 \\
\text { [7] }\end{array}$ & Tapentadol (Nucynta) & No rescue & No Rescue & $\begin{array}{l}\text { 11-point } \\
\text { NPRS }\end{array}$ & 121 & 119 \\
\hline Daniels Casson 2009 [4] & Tapentadol (Nucynta) & $1000 \mathrm{mg}$ APAP & Weak & $\begin{array}{l}\text { 11-point } \\
\text { NPRS }\end{array}$ & 69 & 275 \\
\hline Altman 2013 [1] & $\begin{array}{c}\text { Indomethacin } \\
\text { submicron (Tivorbex) }\end{array}$ & $\begin{array}{c}1^{\text {st: }} 5 \mathrm{mg} \mathrm{HC} / 500 \mathrm{mg} \\
\text { APAP; } 2^{\text {nd: }} 7.5 \mathrm{mg} \text { OC } / \\
325 \mathrm{mg} \text { APAP }\end{array}$ & Liberal & $\begin{array}{l}100 \mathrm{~mm} \\
\text { VAS }\end{array}$ & 94 & 91 \\
\hline Jensen 2013 [41] & $\begin{array}{c}\text { Indomethacin } \\
\text { submicron (Tivorbex) }\end{array}$ & $\begin{array}{c}1^{\text {st: }} 5 \mathrm{mg} \mathrm{HC} / 500 \mathrm{mg} \\
\text { APAP; } 2^{\text {nd: }} 7.5 \mathrm{mg} \text { OC } / \\
325 \mathrm{mg} \text { APAP }\end{array}$ & Liberal & $\begin{array}{l}100 \mathrm{~mm} \\
\text { VAS }\end{array}$ & 94 & 92 \\
\hline Gibofsky 2013 [14] & $\begin{array}{c}\text { Diclofenac submicron } \\
\text { (Zorvolex) }\end{array}$ & $10 \mathrm{mg} \mathrm{HC} / 325 \mathrm{mg}$ APAP & Liberal & $\begin{array}{l}100 \mathrm{~mm} \\
\text { VAS }\end{array}$ & 106 & 109 \\
\hline Singla 2014 [31] & MNK-795 (Xartemis XR) & 400 mg Ibuprofen & Liberal & $\begin{array}{l}\text { 11-point } \\
\text { NPRS }\end{array}$ & 163 & 166 \\
\hline Singla 2015 [32] & MNK-155 & $400 \mathrm{mg}$ Ibuprofen & Liberal & $\begin{array}{l}\text { 11-point } \\
\text { NPRS }\end{array}$ & 203 & 203 \\
\hline
\end{tabular}

Boxes highlighted in blue $=$ no rescue; red $=$ weak rescue; green $=$ liberal rescue

$A P A P$ acetaminophen, $H C$ hydrocodone, $m g$ milligram, $m m$ millimeter, NPRS numerical pain rating scale, $O C$ oxycodone,

$V A S$ visual analog scale

${ }^{a}$ Weak rescue = APAP only; Liberal rescue = moderately potent short acting opioids, opioid/APAP combinations or non-steroidal anti-inflammatories

${ }^{b}$ Number of patients randomized

c When multiple doses the value from the lowest overall daily dose is provided

Table 3 caption was published incorrectly and the corrected table should read as follows:

Table 3 Rescue medication and SPID48 values for placebo

\begin{tabular}{|l|c|c|}
\hline Study & Rescue usage & $\begin{array}{c}\text { Normalized } \\
\text { SPID48 }^{\mathbf{a}}\end{array}$ \\
\hline Daniels Upmalis 2009 & No rescue & 24.6 \\
\hline Daniels Casson 2009 & Weak & 54.1 \\
\hline Altman 2013 & Liberal & 155.8 \\
\hline Jensen 2013 & Liberal & 176.9 \\
\hline Gibofsky 2013 & Liberal & 166.1 \\
\hline Singla 2014 & Liberal & 59.7 \\
\hline Singla 2015 & Liberal & 94.4 \\
\hline
\end{tabular}

Weak $=$ Acetaminophen $\quad$ only, $\quad$ Liberal $=$ moderately potent short acting opioids, opioids/APAP combinations or non-steroidal anti-inflammatories

SPID48 summed pain intensity difference from baseline during the $48 \mathrm{~h}$ after the first dose

Boxes highlighted in blue $=$ no rescue, red $=$ weak rescue; green $=$ liberal rescue

${ }^{a}$ No imputation secondary to rescue
The original article was corrected.

Open Access. This article is distributed under the terms of the Creative Commons Attribution-NonCommercial 4.0 International License (http://creativecommons.org/licenses/ by-nc/4.0/), which permits any noncommercial use, distribution, and reproduction in any medium, provided you give appropriate credit to the original author(s) and the source, provide a link to the Creative Commons license, and indicate if changes were made. 